\title{
Planificación curricular en la enseñanza universitaria y desempeño profesional de egresados en educación
}

\author{
Alan Alain Huaman Auccapuri \\ alan.huaman@unsaac.edu.pe \\ ORCID ID: https://orcid.org/0000-0001-9386-9618 \\ Universidad Nacional de San Antonio Abad \\ Zoraida Loaiza Ortiz \\ zoraida.loaiza@unsaac.edu.pe \\ ORCID ID: https://orcid.org/0000-0002-8034-1993 \\ Universidad Nacional de San Antonio Abad \\ Maricia Ursula Urrutia Mendoza \\ maricia.urrutia@unsaac.edu.pe \\ ORCID ID: https://orcid.org/0000-0003-2142-9159 \\ Universidad Nacional de San Antonio Abad \\ Jaime Cuentas Salas \\ jaime.cuentas@unsaac.edu.pe \\ ORCID ID: https://orcid.org/0000-0002-8697-9234 \\ Universidad Nacional de San Antonio Abad \\ Maria Dolores Velasco Palacios \\ maria.velasco@unsaac.edu.pe \\ ORCID ID: https://orcid.org/0000-0001-6978-4112 \\ Universidad Nacional de San Antonio Abad
}

\section{RESUMEN}

El contexto actual exige que la planificación curricular, sea de gran impacto social, cuyo efecto se evidencia en la formación integral del futuro profesional en educación, por ello requiere adaptarse a los cambios que se van generando en el entorno, teniendo en cuenta los avances tecnológicos y científicos, planteamiento de nuevas tendencias y estrategias didácticas. A lo cual el estudio tuvo por objetivo explicar la influencia de la planificación curricular en la enseñanza universitaria y su pertinencia en el desempeño profesional del egresado en educación. El método se circunscribe en la investigación cuantitativa, con un diseño transversal correlacional causal; respecto a la unidad estudio, estuvo conformado por un total de 1772 egresados de la escuela profesional de educación entre los años 2011 al 2019, seleccionándose a un total de 316 egresados a través del muestreo probabilístico sistemático, a quienes se aplicaron dos cuestionarios de respuesta en escala de Likert, habiendo revisado su validez y confiabilidad. Los hallazgos del estudio evidencian que la planificación curricular influye directamente en el desempeño profesional de los 
egresados en educación, dado por el coeficiente de correlación de Pearson a 0,525 y un valor P significativo, la regresión lineal ha demostrado que la planificación curricular es pertinente y debidamente articulada con el desempeño profesional en un $27,6 \%$ de los egresados en educación, mientras el 72,4\% evidencia dificultades para lograr un adecuado desempeño.

Palabras clave: Enseñanza universitaria, desempeño profesional, planificación curricular 


\title{
Curriculum planning in university teaching and professional performance of graduates in education
}

\begin{abstract}
The current context requires that curricular planning be of great social impact, whose effect is evidenced in the comprehensive training of the future professional in education, therefore it requires adapting to the changes that are generated in the environment, taking into account technological advances and scientists, approach to new trends and teaching strategies. To which the objective of the study was to explain the influence of curricular planning in university education and its relevance in the professional performance of the graduate in education. The method is limited to quantitative research, with a causal correlational cross-sectional design; Regarding the study unit, it was made up of a total of 1,772 graduates of the professional school of education between the years 2011 to 2019 , selecting a total of 316 graduates through systematic probability sampling, to whom two response questionnaires were applied in Likert scale, having reviewed its validity and reliability. The study findings show that curricular planning directly influences the professional performance of education graduates, given by Pearson's correlation coefficient at 0.525 and a significant $\mathrm{P}$ value, linear regression has shown that curricular planning is pertinent and duly articulated with professional performance in $27.6 \%$ of education graduates, while $72.4 \%$ show difficulties to achieve adequate performance.
\end{abstract}

Keywords: University teaching, professional performance, curricular planning.

Artículo recibido: 05 de Mayo 2021 Aceptado para publicación: 20 de Junio 2021 Correspondencia: alan.huaman@unsaac.edu.pe Conflictos de Interés: Ninguna que declarar 


\section{INTRODUCCIÓN}

Situar la adquisición de competencias en el centro de la formación universitaria sintetiza y expresa diferentes cambios que se están produciendo en la educación superior. La profunda transformación de la universidad durante las últimas décadas ha estado articulada por algunos hitos significativos.

Hacer referencia a las competencias en el proceso de formación del futuro profesional, es necesario recordar el contexto de la globalización, en el cual la exigencia de la calidad en la educación superior no es solamente hablar de la universidad norteamericana o europea, donde se encuentran las universidades más prestigiosas del mundo, donde países como Singapur, Japón y China, vienen alcanzando los mismos estándares de calidad universitaria, entonces todo depende de la planificación y organización de las universidades, generando alianzas estratégicas, sobre todo con los gobiernos y entidades no gubernamentales involucradas con la investigación y tecnología.

En el contexto Latinoamericano y peruano, la calidad en la educación superior universitaria aun continua en el proceso de adaptación, debido a que aún se menciona que las universidades tienen calidad educativa, pero debido a la globalización y estándares de calidad educativa, la tendencia se enfoca en que la universidad hace, crea o genera calidad educativa, proceso en el cual la comunidad universitaria debe participar activamente, donde no solamente se debe traducir en la cuantificación de la calidad, sino en la capacidad de respuesta de la universidad como impulsora de la calidad educativa. La universidad en estos últimos 20 años viene afrontando desafíos y demandas, tal es el caso de la calidad educativa universitaria, la competitividad de los egresados, la investigación y la innovación tecnológica, entre los más demandados por la sociedad, caracterizada por altos niveles de inestabilidad, incertidumbre y complejidad, asimismo la implantación de las tecnologías de la información y comunicación, la digitalización y nuevas formas de comunicación en la sociedad, requieren de una universidad con la capacidad de responder a las necesidades sociales, especialmente a un mercado laboral cada vez más exigente, centrado en la competitividad profesional de sus egresados.

Es notorio que las universidades en este Siglo XXI, deben comprender la dinámica competitiva del mercado laboral, en tal sentido, como lograr que la universidad se encuentre a la vanguardia de la velocidad y cambio del conocimiento, la globalización del mercado laboral, la sociedad del conocimiento, la economía del conocimiento, la 
virtualización del saber, nuevas competencias y profesionales competentes (Moscoso \& Hernández, 2015, pp. 141-142). Navarro, Pereira, Pereira, \& Fonseca (2010), señala que la educación es proceso de perfeccionamiento durante la vida del hombre, asociada a su visión y la sociedad, de tal forma posibilita la realización de los ideales y del proceso comunicativo, vinculado con la acción dinámica del estudiante, con otras personas y su entorno. (p.203)

En los párrafos anteriores se ha mencionado el contexto de la educación universitaria, el cual está inmerso en la calidad educativa, cada vez más exigente, articulado con la búsqueda de su continuidad a través de la acreditación, los desafíos y exigencias del Siglo XXI, enmarcado en la dinámica competitiva del mercado laboral, se articula y ejecuta a través del proceso educativo formal, cuya esencia estar en manos de la concepción que se tenga del hombre y la sociedad que se desea formar. Justamente para que los fines de la educación se materialicen, es necesario el «currículo» como estrategia filosófica, en un primer momento definida por el positivismo, actualmente aún persiste este paradigma, considerando al hombre como un ser complejo activo, vinculado con la dimensión social y emocional, asimismo con su contexto y su tiempo, conforme se tenga la idea de este tipo de hombre, consecuentemente se concebirá la sociedad que se desee formar.

Las exigencias de la sociedad, políticas educativas planteadas por el Estado y las universidades se materializan gracias al currículo, en efecto es la estrategia por excelencia para lograr los fines educativos, direccionado a satisfacer las necesidades del entorno. No obstante, el «diseño de un plan curricular» es fundamental para poner en marcha los lineamientos curriculares del Estado y las instituciones educativas, articuladas y ajustadas a las necesidades de la sociedad, a través de la responsabilidad conformada por una gerencia académica.

En el sector universitario la planificación es una función permanente de reajustes en todo el proceso educativo y curricular, con el fin de responder a las demandas sociales y laborales, enfocado en una formación profesional por competencias, donde toda acción está sujeta a una previsión, denominada «planificación del currículo o curricular», orientado a la elaboración e implementación de un plan curricular, donde se establece los lineamientos necesarios para el proceso formativo del estudiante universitario, plasmado en un documento normativo, visible y evaluable, contempla los objetivos, competencias profesionales, malla curricular, sumillas y distribución de los créditos según 
competencias genéricas o transversales y específicas, lo cual viene a conformar el plan operativo de los aprendizajes por cada asignatura.

El principal problema observado en las universidades peruanas, más aún en la nacionales, es la insatisfacción por parte de los egresados de las diferentes carreras, programas o escuelas profesionales, debido a la falta de eficacia en el desempeño profesional de los egresados, como es el caso de los egresados en educación, quienes reciben una formación profesional mayormente teórica, que muchas veces no responde a la actividad profesional, debido a los planes curriculares a pesar de contar con los elementos propicios para generar una formación teórica y práctica, muchas veces solo queda plasmada en un documento, en este panorama se fundamenta la necesidad de conocer si la planificación curricular esta articulada y responde a un adecuado desempeño profesional por parte de los egresados en educación.

En atención a lo planteado, el estudio hace notorio la necesidad de una planificación curricular, que debe estar estrechamente vinculado y articulado con la formación profesional del estudiante, evidenciando el grado de su efectividad a través del desempeño profesional del egresado, en este caso se enfoca en los profesionales en educación, egresados de las Facultades de Educación o Pedagogía, caso particular de los egresados de la Escuela Profesional de Educación, Facultad de Educación y Ciencias de la Comunicación de la Universidad Nacional de San Antonio Abad, de la provincia y departamento del Cusco, República del Perú, de esta forma se tiene el siguiente problema: «¿Cuán adecuado es la planificación curricular en la enseñanza universitaria y su pertinencia en el desempeño profesional del egresado en educación?»

Para responder apropiadamente a esta interrogante se ha recurrido a los estudios realizados por diferentes autores, tales como el «El proceso de elaboración del currículo para la formación profesional universitaria» escrito por Mejía (2011), enfocándose en la comprensión de un currículo integral, constituido por las capacidades del hombre: lenguaje, pensamiento, habilidades mecánicas y políticas, a partir de lo cual se propone una metodología que responda a la formación profesional universitaria, teniéndose en cuenta el diagnóstico de la escuela profesional, el perfil profesional real, campo o área de desempeño laboral, proceso de diseño curricular, organización estructural del currículo (rigidez y flexibilidad), plan de estudios (malla curricular), sumillas y sílabos, vienen a conformar el proceso que se debe seguir para una adecuada articulación a las necesidades 
del mercado laboral, respondiendo al desarrollo de las competencias especificas y genéricas que conformar parte del plan de estudios.

El estudio «incorporación del concepto de competencia en el diseño curricular universitario» escrito por Larraín, González, \& Espinosa (2010), en sus comentarios finales mencionan los cambios en el diseño curricular y la docencia universitaria, donde la formación por competencias, debe centrarse en los aprendizajes y no solamente en la enseñanza, valorando los aprendizajes previos, donde los docentes universitarios deben caracterizarse por una formación en principios, herramientas e implicaciones del enfoque por competencias, inciden también en la importancia por la «relación entre la teoría y la práctica, que le permita a los estudiantes contextualizar los conocimientos adquiridos. Ello se logra a través de diferentes metodologías de enseñanza (análisis de casos, salidas a terreno, elaboración de proyectos y prácticas tempranas en terreno)» debiéndose vincular en los procesos de panificación curricular en respuesta al sector productivo y demanda laboral; los procesos de evaluación deben responder a garantizar el dominio de los aprendizajes, a través de la implementación de instrumentos adecuados, tales como ejercicios de simulación o casos directamente relacionados con la práctica, debiendo contar cada instrumento con la validez del caso (miden lo que se quiere medir) y confiables y consistentes (devuelven los mismos resultados para poblaciones diferentes), es decir, los instrumentos presentan la capacidad de repetibilidad en distintos contextos, lo cual conlleva a modificar los sistemas de calificación, debiendo cambiar el clásico sistema de promedios por un listado de competencias que el estudia debe dominar.

En ese entender el estudio, en respuesta al problema formulado, tiene por objetivo: «Explicar la influencia de la planificación curricular en la enseñanza universitaria y su pertinencia en el desempeño profesional del egresado en educación», a partir de la revisión de la literatura relacionada con las teorías curriculares de la planificación curricular en el contexto de la enseñanza universitaria y la importancia por la articulación y respuesta apropiada a las exigencias del desempeño profesional del egresado en educación de la Escuela Profesional de Educación, Facultad de Educación y Ciencias de la Comunicación de la Universidad Nacional de San Antonio Abad, caracterizado por la implementación en los últimos 4 años por un plan de estudios por competencias, esta característica está estrechamente vinculada con las exigencias de la nueva Ley Universitaria 30220, orientado a garantizar la calidad educativa universitaria del Perú. 


\subsection{Planificación curricular y desarrollo de la enseñanza universitaria}

El currículo engloba los propósitos, procesos y objetivos compartidos por una sociedad. Hace referencia a una política, un proceso de transferencia, comunicación y generación de conocimientos, puntos que se desarrollan en el contexto educativo, por tanto, el currículo es en sí planificación. (Bastidas, 2019)

La planificación curricular hace referencia a la implantación de la «metodología científica» para darle solución a problemas prácticos «índole instruccional», con el fin de guiar el actuar educativo y ejecutarla con eficiencia. Se toma en cuenta además la conexión entre la posibilidad de regular el procedimiento instruccional (enseñanzaaprendizaje) y la planificación curricular, que de forma peculiar se desarrollan en el contexto formativo. (Navarro, Pereira, Pereira, \& Fonseca, 2010)

Por su parte Kaufman (2011), afirma que: «Un enfoque sistemático educativo direccionado al accionar educativo, misma que necesita que se lleve a cabo una planificación sistemática y formal, al igual que las aplicaciones, diseños, análisis y evaluaciones» (p.14).

Bastidas (2019), menciona que el currículo es solicitante y a su vez productor de política. Además, esta advierte las permutas e innovaciones necesarias por la sociedad educativa y sus integrantes (dimensión evaluativa de la política) en todos los contextos; por otro lado, esta es responsable de formar a los integrantes educativos para que estos lleven a cabo y dinamicen tales permutas.

De acuerdo con Compte (2013), el proceso de planificación curricular en el ámbito de la educación superior engloba distintas fases: «análisis previo, diseño curricular, aplicación y evaluación curriculares», en la segunda fase mencionada se detallan los propósitos educativos esto con sustento en la primera; se diseñan medios (procesos y contenidos), se fija el talento humano, recursos financieros e informativos, para de esta forma alcanzar los objetivos establecidos.

En la educación superior, la planificación curricular pretende conseguir un funcionamiento pleno de sus especialidades, y estas deben proporcionar resolución a los conflictos a nivel educativo y formativo, además de aquellas de índole política, económica y social; y de esta forma los planes de estudio se hallen contextualizados, actualizados y sujetas a las tendencias diciplinares, y se hallen acorde a los requerimientos del mercado laboral en el cual se desenvuelven los egresados. 
Bastidas (2019), afirma además que todo currículo debe hallarse orientado a conseguir el perfil que se pretende de los profesionales en formación, misma que debe estar sujeta a la demanda actual de la sociedad globalizada, cambiante, interdependiente y compleja; la implantación de factores tecnológicos debe estar direccionada a una formación integral del egresado. Se menciona además que el currículo es agente de comunicación entre las sinergias que interactúan en el contexto educacional y sus actores.

\subsubsection{El currículo universitario}

Tobón, Pimienta, \& García (2010), afirman que el diseño curricular universitario es una actividad inherente a la docencia universitaria, misma que se desarrolla en el marco de los proceso colaborativos, y pretende colaboración de todos los actores de la comunidad universitaria, y de esta forma se labore en base a una visión compartida, con lo cual los procedimientos académicos abarquen los diferentes retos educacionales; este proceso considera la dinámica cultural y social de la institución, y esta se lleva a cabo a través del «trabajo en equipo»y siendo el fin lograr una formación integral. «La construcción del currículo debe ser un macroproyecto formativo que se halle en contante mejora y garantice una formación acorde a los estándares y los requerimientos del actual contexto» (Tobón, Pimienta, \& García, 2010, p.5)

El currículo de un programa universitario busca esencialmente proporcionar al estudiantado habilidades, capacidades, competencias necesarias para su buen desenvolvimiento profesional. Es un sistema que se sustenta en aspectos culturales y sociales, además de la lucha de "poder-saber» que se dan en el contexto actual y se establecen en diseños particulares dentro de las universidades. El currículo debe sujetar una vinculación abierta con la sociedad, el contexto y las personas. (Litwin, 2006)

\subsubsection{Plan de estudios universitario}

Para Zabalza (2000), el plan de estudios establece el procedimiento de formalización de distintas decisiones curriculares de organización de los conformantes (asignaturas, seminarios, módulos, talleres, etc.), acogidas en función a diversos criterios claramente fijados. Las competencias específicas y genéricas son la base de la elaboración del plan de estudios; seguidamente debe fijarse el número de elementos curriculares a lo largo de la formación profesional, concierne la formación específica, general y especializada, añadiendo las practicas preprofesionales como elementos curriculares, misma que direccione a una formación integral y responsable. 
Tobón (2008), afirma que: «El plan de estudios se denomina como proyecto formativo integrado, que profiere estrategias, mismas que se emplean para dar solución a problemas contextualizados en la realidad y en sus cambios constantes» (p.11).

Además, el autor en mención afirma que los proyectos formativos poseen los siguientes objetivos esenciales:

- Aprende a entender y a estructurar la realidad, como un «tejido problémico», que surge por la constante organización, orden-caos, con reconocimiento y afrontamiento estratégico de la incertidumbre.

- Realizar una formación sistemática de competencias a través de la integración del «saber hacer», con «el saber» y el «saber ser».

- Conocer, entender y dar solución a conflictos de la realidad paralela a los intereses del estudiantado.

\subsubsection{Currículo por competencias}

Catalano, Avolio, \& Sladogna (2004), mencionan que el diseño curricular basado en competencias es desarrollado en base a lo detallado en el perfil profesional, con el cual el desenvolvimiento de un individuo en un determinado contexto debe hallarse acorde a los roles establecidos en esta y a su formación. Si el perfil establecido se halla sujeta a la realidad y a la demanda del contexto laboral la posición de empleabilidad de los egresados es mayor.

Por su parte González (2006), menciona que en el ámbito de la educación superior se requiere especificar que tipología especifica de competencias laborales son las competencias profesionales; es decir, las capacidades vinculadas al desempeño eficiente de la labor profesional. «Las competencias profesionales son eficaces cuando abarcan capacidades, conocimientos, actitudes y valores; estas cobran sentido cuando se evidencian en el desarrollo de una actividad laboral específica, para esta existen criterios de consecución, calidad y efectividad ya determinadas» (p.14).

En este entender un currículo universitario basado en competencias no solo se vincula al ámbito laboral, sino que también se enfoca en el individuo, en su integridad, como ser de desarrollo e integrante social. Si bien las competencias laborales avalan un desempeño profesional determinado, no cubren del todo la formación del egresado tanto a nivel personal y social. En este entender la formación personal, para la producción y la labor, además para la formación de convivencia en sociedad pueden establecerse ejes esenciales 
de cualquier acto educativo que se lleve a cabo en la educación superior en general y en especial en la universitaria.

\subsection{Desempeño profesional}

Carrera, Parreño, \& Ayala (2018), afirma que el desempeño profesional hace referencia a todas las acciones llevadas a cabo por una persona que aprendió una profesión u oficio, que se puede dar por un proceso formativo o basado en la experiencia; tal desempeño debe mostrar habilidades, conocimientos e idoneidad en la realización de labores, estén estas vinculadas a la producción de bienes o prestación de servicios.

El desempeño profesional como actividad llevada a cabo por una persona en el desempeño profesional o actividad aprendida, que le faculta a demostrar su habilidad para dar solución a problemas.

Añorga (2014), por su parte la considera como una capacidad persona para llevar a cabo determinadas tareas, responsabilidades y deberes inherentes a las funciones asignadas de su puesto de labor. El desempeño profesional se manifiesta en el modo de actuar y en la ejecución de labores por parte de una persona en el cumplimiento de su papel dentro de una organización.

Carrera, Parreño, \& Ayala (2018), asevera que el desempeño profesional cumple un rol relevante, debido a que esta es ejecutada por las personas, que, dentro de una organización, entidad pasa a formar parte del talento humano, tal ejecución se lleva a cabo con el manejo de conocimientos técnicos y conforman una variable requerida para cuantificar la eficacia de la producción o prestación dentro de una organización.

En base a lo mencionado es prudente resaltar que una profesión no siempre se adquirirá de manera técnica, científica; sino que este proceso puede darse por un aprendizaje apoyado en la experiencia; en ambos casos el aspecto relevante es que en el cumplimiento de las funciones encomendadas el desempeño profesional de una persona este acorde a los requerimientos de la organización y a las exigencias de la sociedad.

\subsubsection{Mejoramiento del desempeño profesional}

Añorga (1999), afirma que existen ciertas predisposiciones respecto a la mejora del desempeño profesional:

- Esta se vincula al desarrollo de las competencias profesionales, que es consustancial al puesto o funciones del talento humano. 
- Para una mejora continua y la adaptación a las demandas actuales se hace necesaria la preparación, superación, capacitación de las personas.

- La mejora esta direccionada a incrementar la calidad del desempeño profesional de las personas.

En base a los puntos mencionados, el desempeño profesional es el procedimiento que se apoya en el constante aprendizaje y adaptación a los cambios, además de desarrollarse en una estructura de relaciones e interrelaciones, de connotación social determinadas entre los integrantes inmersos en una organización.

Por su parte Medrano \& Molina (2010), afirma que la mejora del desempeño profesional es viable siempre y cuando los programas de formación continua se direccionen a la estructuración de competencias profesionales, mismas que contribuyan a un mejor desenvolvimiento de las personas. Adoptar un «referencial por competencias» evidencia la intencionalidad de implantación de una figura «practica reflexiva», siendo este eje de todo planteamiento formativo. En el caso específico del contexto educativo se hace notoria la permuta de una perspectiva de un profesional de la educación que se hace cargo, crítica y conscientemente de la estructuración de modelos, métodos, estrategias y de un currículo, que apoyen a la mejora la de enseñanza siendo este uno de los fines del desempeño profesional de un educador.

Para un óptimo desempeño profesional de un individuo no solo basta con la preparación teórica y la adquisición de conocimientos, si no que este a su vez debe ser competente y debe estar en constante aprendizaje y actualización. Los conocimientos prácticos requieren de la consecución de capacidades, destrezas, habilidades y procesos respecto a los instrumentos, técnicas e innovaciones que se empleen para la realización de actividades, de manera tal que se optimice la calidad del desempeño.

\subsubsection{El desarrollo de competencias para el desempeño profesional}

Medrano \& Molina (2010), mencionan que las competencias profesionales tienen conexión con distintas particularidades con las que cuenta un profesional, las cuales le posibilitan convivir e interactuar en un determinado contexto. La competencia se sustenta en las capacidades individuales, y para analizarlas es esencial posicionarla en distintos ámbitos interrelacionados: 
- En un ámbito especifico, conformado por el conocimiento de las distintas áreas diciplinares particulares de una profesión, como también de las destrezas y habilidades propias a la praxis profesional.

- La perspectiva personal, con engloba las actitudes, posturas, intereses y expectativas.

- El campo social-contextual, concerniente al conocimiento y entendimiento del contexto, además del vínculo e interacción social, mismos que son aspectos relevantes para la adaptación personal.

La competencia es comprendida como un conglomerado de saberes, capacidades, habilidades, conductas, aptitudes y actitudes concertadas que se emplean para la solucionar problemas que se presenten en el ejercicio de una profesión, con lo cual una persona demuestra su «saber ser y estar» en un lugar y momento determinado, lo cual le posibilita reaccionar oportunamente frente a los requerimientos y desafíos que se presenten, aun así, estos sean complejos y cambiantes.

\subsubsection{Evaluación de la competencia y el desempeño profesional}

De acuerdo con Salas, Hatim, \& Rey (1997), la competencia hace referencia a la capacidad de ejecutar una labor de manera adecuada. Es un conglomerado de habilidades y conocimientos esenciales, como también de actitudes y aptitudes de las personas, «es lo que sabe y sabe hacer». En tanto el desempeño hace referencia a la conducta real del individuo para llevar a cabo competentemente sus funciones o roles propios de su ámbito de labor, «es lo que en realidad hace».

Específicamente el termino de evaluar, hace referencia a la emisión de un «juicio de valor» respecto a una labor o acción determinada, esto como producto de la comparación de un escenario observado y un patrón predeterminando. Con lo cual es factible cuantificar variables de desempeño y consecuentemente en base a ellos se procesa a una toma de decisiones.

La evaluación de la competencia y el desempeño profesional se puede desarrollar con dos propósitos:

- Con fines diagnósticos, para evaluar la calidad de aprendizaje y eficacia del sistema educacional formativo, o para reconocer los requerimientos de aprendizaje.

- Con fines certificativos, sean estos de carácter académico (asignación, curso, entrenamiento, etc.) o para la autorización legal de ejercicio profesional o especialista. 


\section{MATERIALES Y MÉTODOS}

El estudio recabó datos transversales de un total 1772 egresados de la Escuela Profesional de Educación, Facultad de Educación y Ciencias de la Universidad Nacional de San Antonio Abad, provincia y departamento del Cusco, cuyas edades fluctúan en su mayoría entre los 26 a 30 años que viene a ser el 46,2\%, de 20 a 25 años el 27,8\% y de 31 años más el 25,9\%; en su mayoría son jóvenes egresados, siendo seleccionados de acuerdo a la información obtenida de la Unidad de Estadística de la Universidad Nacional de San Antonio Abad, específicamente de la publicación denominada «variables estadísticas de los años 2011 al 2019» y es justamente en esta población fue seleccionada para la recolección de la información, por haber egresado recientemente recuerdan con mayor facilidad el proceso de su formación profesional, pudiendo brindar información más confiable.

El método del estudio se fundamenta en la investigación cuantitativa, de tipo explicativo, orientada a la búsqueda de las circunstancias que determinan el comportamiento de la variable dependiente, que en este caso es la influencia de la planificación curricular en la pertinencia del desempeño profesional del egresado en educación, como los datos fueron recolectados en una sola ocasión responde al diseño transversal correlacional causal, asimismo no existe ninguna manipulación o intervención por parte de los investigadores, se ejecutó el análisis estadísticos bivariado para determinar la correlación, porque se realizó la cuantificación de las variables planificación curricular y desempeño profesional, lo cual permitió realizar los procedimientos estadístico respectivos. (Hernández \& Mendoza, 2018)

La unidad muestral obtenida fue a través del muestreo probabilístico sistemático, debido la considerable cantidad de egresados, procediéndose previamente al cálculo del muestre probabilístico al azar simple, obteniéndose $n=315,852 \approx 316$, teniendo en cuenta como margen de error el 0,05, desviación estándar de $\sigma=0,5$, el nivel de confianza de 95\%, distribución normal estándar de acuerdo al nivel de confianza $Z= \pm 1,96$, habiéndose obtenido por aproximación la cantidad de 316 egresados, quienes fueron distribuidos por especialidad de acuerdo a la información proporcionada por el Centro de Cómputo de la Universidad Nacional de San Antonio Abad para recabar información más valiosa y representativa, distribuida de la siguiente manera: 
Tabla 1. Estadísticos descriptivos de los egresados seleccionados en la muestra por especialidad

\begin{tabular}{lcc}
\hline \hline \multicolumn{1}{c}{ Especialidad } & Cantidad & Porcentaje \\
\hline Ciencias Naturales & 65 & $20,6 \%$ \\
Ciencias Sociales & 48 & $15,2 \%$ \\
Educación Física & 25 & $7,9 \%$ \\
Educación Primaria & 69 & $21,8 \%$ \\
Lengua y Literatura & 51 & $16,1 \%$ \\
Matemática y Física & 58 & $18,4 \%$ \\
Total & 316 & $100,0 \%$ \\
\hline \hline
\end{tabular}

Nota: Resultados procesados en el programa estadístico IBM SPSS.

Los instrumentos aplicados para recolectar la información fueron dos cuestionarios debidamente validados, que responde a la técnica de la encuesta, para la recolección de la variable planificación curricular se utilizó el «Cuestionario de planificación y desarrollo de la enseñanza universitaria - Satisfacción de los estudiantes», cuyos autores son Fdo. Rosa María Fernández Alcalá, Vicedecana de Calidad de la Facultad de Ciencias de la Salud de la Universidad de Jaén, de fecha 10/06/2014, en el caso de la variable desempeño profesional se utilizó el «Cuestionario de desempeños profesionales de profesores en pro de una educación creativa: evaluación y autoevaluación desde la perspectiva docente», cuyo autor es Marco para la buena dirección perteneciente al Ministerio de Educación de Chille del año 2015. Ambos instrumentos cuentan con preguntas cerradas en escala Likert de 1 a 5 puntos, ajustado de acuerdo al contexto social y cultural de los egresados de la Escuela Profesional de Educación, en ambos casos el número de ítems fue de 20, pero primigeniamente se tenia 28 y 23 ítems respectivamente, posteriormente a la validación se realizó la reducción del caso porque algunos ítems eran inconsistentes, donde los resultados del Índice de Consistencia Interna Alfa de Cronbach fueron para el cuestionario de planificación curricular $\alpha_{1}=0,807$ y el cuestionario de desempeño laboral $\alpha_{2}=0,824$ de tal manera, ambos representan datos fiables $\mathrm{y}$ consistentes, teniendo en cuenta la reducción realizada mencionada líneas más atrás. El procesamiento de los resultados fue considerando las características y tipo de datos obtenidos, vale decir, el estudio consideró la sumatoria de la información obtenida, la cual corresponde al tipo de dato discreto o rango, que viene de sumar las respuestas de los egresados según el total de ítems y dimensiones, para la elaboración de la matriz de datos 
se utilizó el programa Microsoft Excel y en el caso de los procedimientos estadísticos se utilizaron el programa estadístico Minitab y IBM SPSS.

La presentación de los datos estuvo dado por la estadística descriptiva, para variables de tipo discretos, es decir, se calculó la media, varianza y desviación estándar se cuerdo a la necesidad del objetivo del estudio, representado gráficamente en diagramas de cajas y bigotes segmentado por especialidad, edad o tiempo de servicios profesionales, posteriormente se realizó el análisis inferencial (estadística inferencial) mediante el estadígrafo correlacional lineal de Pearson y adicionalmente el análisis de la regresión lineal (análisis multivariado), teniendo en cuenta la determinación de los supuestos de normalidad para estadígrafos paramétricos como es el caso de la correlacional lineal de Pearson. (Johnson \& Kuby, 2008)

La medición de las variables fue posible a través del siguiente cuadro de operacionalización de variables:

\begin{tabular}{|c|c|c|}
\hline Variables & Dimensiones & Indicadores \\
\hline \multirow{4}{*}{$\begin{array}{l}\text { Planificación } \\
\text { curricular }\end{array}$} & $\begin{array}{l}\text { Objetivos del plan de } \\
\text { estudios }\end{array}$ & $\begin{array}{l}\text { - } \text { Claridad de los objetivos. } \\
\text { - Satisfacción con los objetivos. }\end{array}$ \\
\hline & $\begin{array}{l}\text { Planificación de la } \\
\text { enseñanza }\end{array}$ & $\begin{array}{l}\text { - } \quad \text { Desarrollo del plan de estudios. } \\
\text { - } \text { Implementación de sílabos. } \\
\text { - Planificación de las asignaturas. }\end{array}$ \\
\hline & Desarrollo de la enseñanza & $\begin{array}{l}\text { - } \quad \text { Desarrollo de la enseñanza. } \\
\text { - Métodos de enseñanza. }\end{array}$ \\
\hline & Evaluación de aprendizajes & $\begin{array}{l}\text { Instrumentos de evaluación. } \\
\text { - Evaluación de competencias. }\end{array}$ \\
\hline \multirow{5}{*}{$\begin{array}{l}\text { Desempeño } \\
\text { profesional }\end{array}$} & Autonomía profesional & $\begin{array}{l}\text { - Iniciativa pedagógica. } \\
\text { - Capacitación en cursos y talleres. }\end{array}$ \\
\hline & $\begin{array}{l}\text { Apoyo a los estudiantes en } \\
\text { su formación escolar }\end{array}$ & $\begin{array}{l}\text { - Estrategias y recursos para generar } \\
\text { creatividad. } \\
\text { - Propuestas y/o proyectos para } \\
\text { promover la creatividad. }\end{array}$ \\
\hline & $\begin{array}{l}\text { Practicas pedagógicas } \\
\text { reflexivas }\end{array}$ & $\begin{array}{l}\text { Estimular el pensamiento crítico } \\
\text { en aula. } \\
\text { - Enseñanza creativa en aula. }\end{array}$ \\
\hline & $\begin{array}{l}\text { Relaciones con los padres de } \\
\text { familia }\end{array}$ & $\begin{array}{l}\text { - Orientación a los padres de } \\
\text { familia. } \\
\text { - Motivar la participación de los } \\
\text { padres de familia. }\end{array}$ \\
\hline & $\begin{array}{l}\text { Relaciones profesionales } \\
\text { entre directivos y docentes }\end{array}$ & $\begin{array}{l}\text { - Relaciones interpersonales entre } \\
\text { docentes. } \\
\text { - Integración para trabajar equipo }\end{array}$ \\
\hline
\end{tabular}

Nota: Adaptación realizada en base a la revisión del estudio realizado por Navarro, Pereira, Pereira, \& Fonseca (2010) respecto a la planificación curricular en la enseñanza universitaria de calidad y Añorga (2014) en relación con el desempeño profesional de los egresados universitarios en educación. 


\section{RESULTADOS Y DISCUSIÓN}

\subsection{Resultados}

La percepción que tienen los egresados respecto a la forma en que se desarrolla la planificación curricular en el proceso de formación profesional, de acuerdo al género y tiempo de servicios, presentan lo siguiente resultados, a lo cual previamente se ha realizado los análisis descriptivos para variables discretas.

Tabla 2. Estadísticos descriptivos de la variable educación financiera y sus dimensiones

\begin{tabular}{lcccc}
\hline \hline \multicolumn{1}{c}{ Dimensiones / Variable } & n & Media & $\begin{array}{c}\text { Desviación } \\
\text { estándar }\end{array}$ & Varianza \\
\hline Objetivos del plan de estudios & 316 & 15,56 & 2,841 & 8,069 \\
Planificación de la enseñanza & 316 & 16,33 & 3,209 & 10,299 \\
Desarrollo de la enseñanza & 316 & 18,55 & 3,388 & 11,480 \\
Evaluación de aprendizajes & 316 & 17,50 & 3,164 & 10,010 \\
Planificación curricular & 316 & 67,94 & 9,971 & 99,428 \\
\hline \hline
\end{tabular}

Nota: Datos procesados en el programa estadístico IBM SPSS.

Los hallazgos indican una dispersión relativamente homogénea en las dimensiones, dando a entender que los objetivos del plan de estudios, planificación de la enseñanza, desarrollo de la enseñanza y evaluación de aprendizajes, es percibida de forma divergente en cuanto al desarrollo de la planificación curricular, en cuanto a las medias dan a entender que está debidamente estructurada, demostrándose la probabilidad de una adecuada implementación del plan de estudios, que responde a las necesidades educativas del estudiante de la escuela profesional de educación, respondiendo a una formación profesional debidamente planificada y coherente con los objetivos a lograr.

Tabla 3. Estadísticos descriptivos de la variable desempeño profesional y sus dimensiones

\begin{tabular}{lcccc}
\hline \hline \multicolumn{1}{c}{ Dimensiones / Variable } & $\mathbf{n}$ & Media & $\begin{array}{c}\text { Desviación } \\
\text { estándar }\end{array}$ & Varianza \\
\hline Autonomía profesional & 316 & 13,03 & 2,957 & 8,742 \\
Apoyo a los estudiantes en su & 316 & 13,10 & 2,604 & 6,783 \\
formación escolar & 316 & 13,50 & 3,143 & 9,876 \\
Practicas pedagógicas reflexivas & 316 & 12,66 & 2,779 & 7,725 \\
Relaciones con los padres de familia & 316 & 13,89 & 3,450 & 11,899 \\
Relaciones profesionales entre & 316 & 66,17 & 11,299 & 127,660 \\
directivos y docentes & Desempeño profesional &
\end{tabular}

Nota: Datos procesados en el programa estadístico IBM SPSS. 
Los resultaos de la dispersión muestran relativa similitud, lo cual denota divergencia o distintos puntos de vista en cuanto al desempeño profesional que vienen experimentando, en tanto, que probabilísticamente es posible determinar dificultades durante el accionar profesional de los educadores, pues la media confirma esta situación porque no se observan promedios alentadores, que si tiene la planificación curricular. Los profesionales en educación se enfrentan a una realidad laboral en la cual no cuentan con la debida experiencia práctica y poco desarrollo de sus competencias profesionales al momento de desplegar su autonomía profesional, apoyo a los estudiantes en su formación escolar, practicas pedagógicas reflexivas, relaciones con los padres de familia y relaciones profesionales entre directivos y docentes. Desde ya se puede observar que muchas veces lo desarrollado en las aulas universitarias, no responde con las capacidades y habilidades necesarias para un adecuado desempeño profesional del egresado en educación.

Veamos a continuación como se visualiza dicho comportamiento, de acuerdo a lo señalado en las tablas anteriores.

Figura 1. Diagrama de cajas y bigotes de la planificación curricular por tiempo de servicios profesionales de los egresados en educación

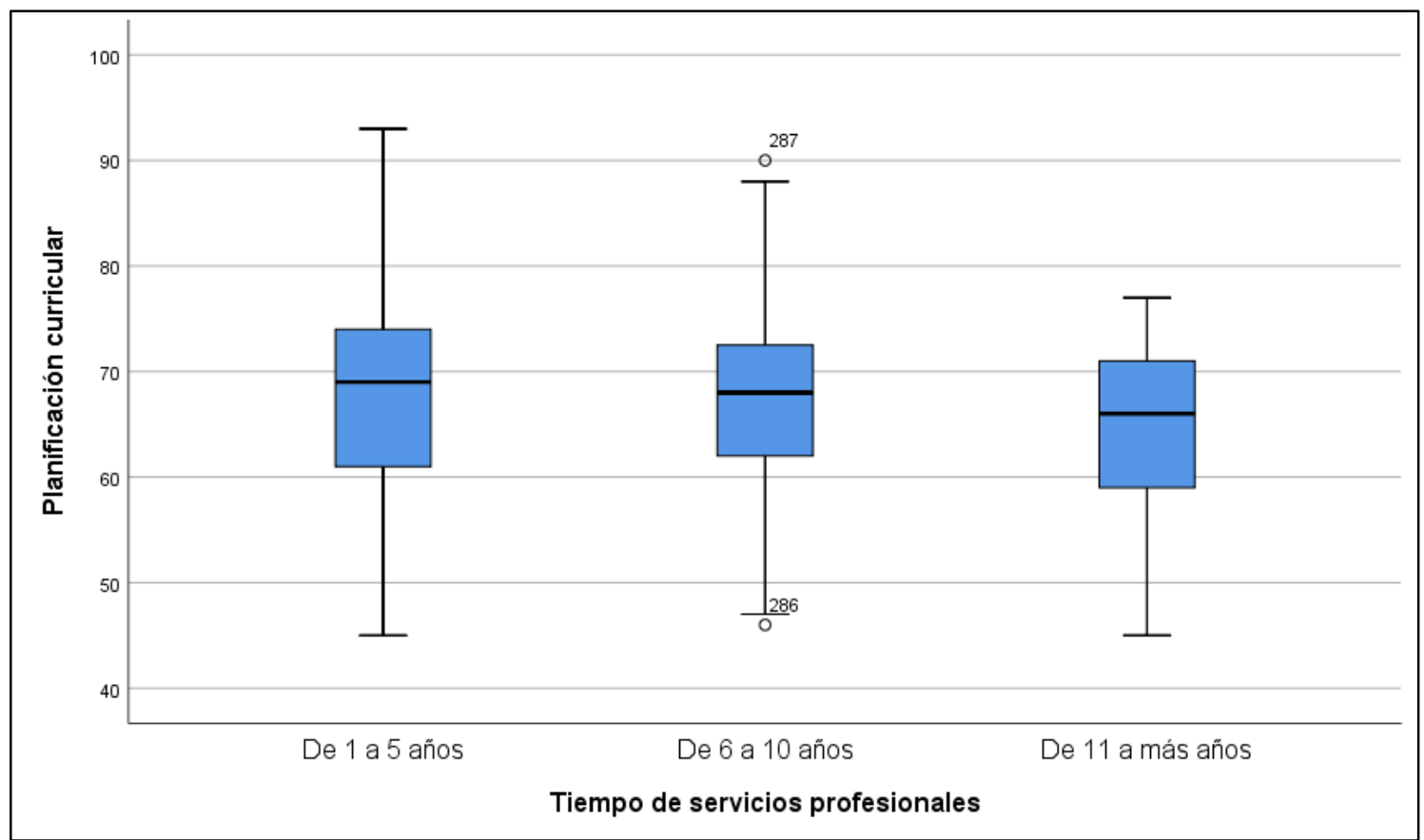

Nota: Datos procesados en el programa estadístico IBM SPSS de un total de 316 egresados en educación.

La planificación curricular visualizada mediante diagramas de cajas y bigotes se acuerdo al tiempo de servicios profesionales, evidencia un fuerte aceptación y positiva 
estructuración de los objetivos del plan de estudios, planificación de la enseñanza, desarrollo de la enseñanza y evaluación de aprendizajes en los primeros 5 años de servicios profesionales, a medida que pasa el tiempo, esta percepción va decayendo, demostrando que la planificación curricular no responde eficazmente conforme la actividad laboral demanda un mejor desempeño, dado por las nuevas tendencias y exigencias del Ministerio de Educación, instituciones privadas y afines al desarrollo de la actividad educativa. A continuación, veamos que ocurre con el desempeño profesional.

Figura 2. Diagrama de cajas y bigotes del desempeño profesional por tiempo de servicios profesionales de los egresados en educación

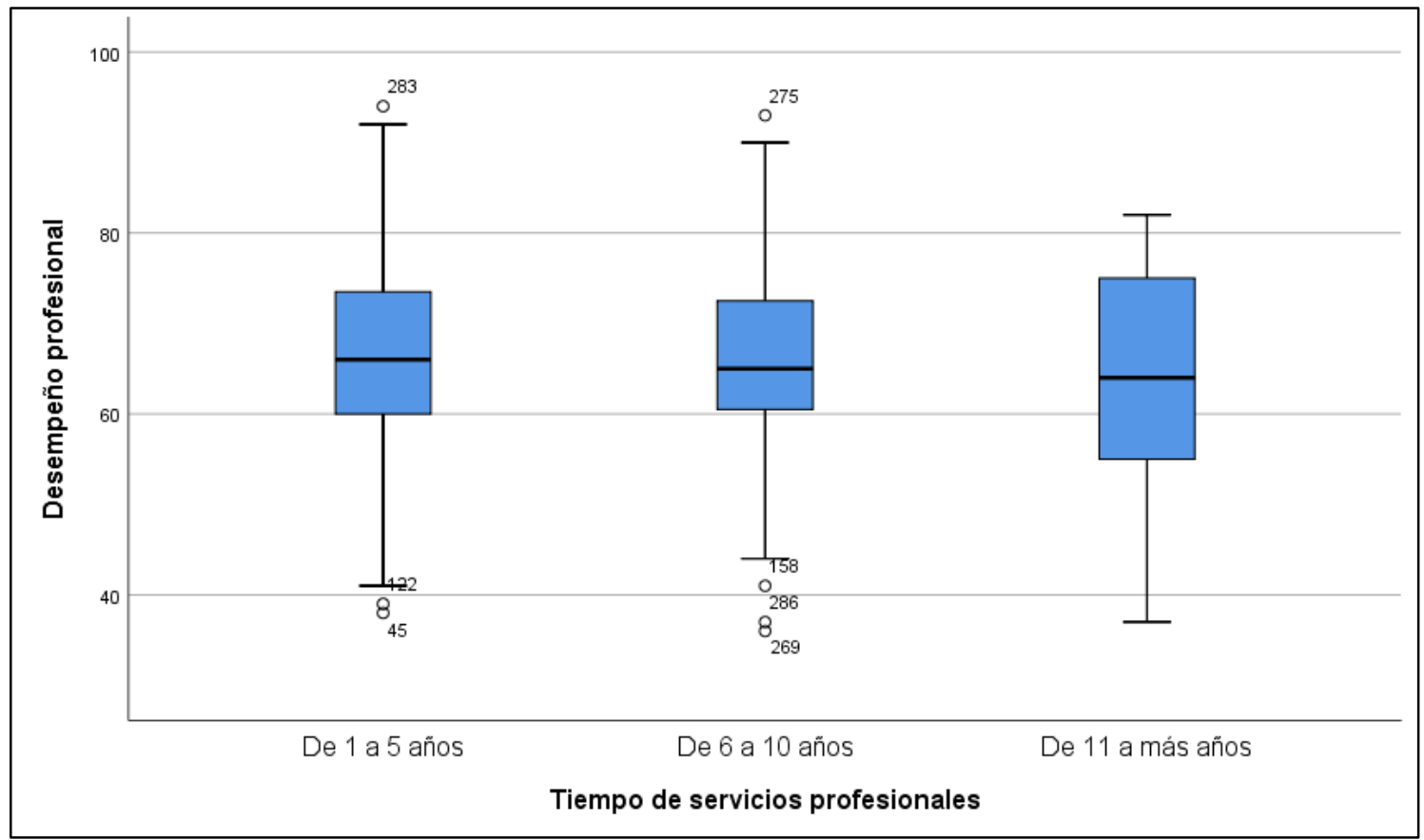

Nota: Datos procesados en el programa estadístico IBM SPSS de un total de 316 egresados en educación.

El desempeño profesional de los egresados en educación muestra un comportamiento distinto a la planificación curricular, debido a que en los primeros años de trabajo presentan dificultades para desarrollar y consolidar su autonomía profesional, apoyo a los estudiantes en su formación escolar, practicas pedagógicas reflexivas, relaciones con los padres de familia y relaciones profesionales entre directivos y docentes, conforme el tiempo pasa más allá de los 10 años, por la propia necesidad laboral, propia en ellos la necesidad de enfocarse en asistir o inscribirse a diversas capacitaciones para aprender aquellos conocimientos prácticos que no desarrollaron en las aulas universitarias. Es evidenciable que después de los primeros 5 años de servicios profesionales, algunos logran desempeñarse mejor a pesar de las circunstancias, mientras que algunos empeoran 
en afianzar las destrezas necesarias o les toma más tiempo poder acomodarse a las exigencias laborales.

Para proceder con el análisis de la correlación, es necesario demostrar la normalidad en las variables planificación curricular y desempeño profesional.

Tabla 4. Estadístico de Kolmogorov-Smirnov para determinar la normalidad de los datos de las variables planificación curricular y desempeño profesional

\begin{tabular}{lcc}
\hline \hline Estadísticos & $\begin{array}{c}\text { Planificación } \\
\text { curricular }\end{array}$ & $\begin{array}{c}\text { Desempeño } \\
\text { profesional }\end{array}$ \\
\hline $\mathrm{n}$ & 316 & 316 \\
Estadístico de prueba & 0,044 & 0,046 \\
Sig. asintótica(bilateral) & $0,200^{\mathrm{c}, \mathrm{d}}$ & $0,200^{\mathrm{c}, \mathrm{d}}$ \\
\hline \hline
\end{tabular}

a. La distribución de prueba es normal.

b. Se calcula a partir de datos.

c. Corrección de significación de Lilliefors.

d. Esto es un límite inferior de la significación verdadera.

Los resultados del estadístico Kolmogorov-Smirnov, de acuerdo a lectura del valor P o significancia asintótica bilateral, resulta ser mayor al nivel de significancia del 5\%=0,05, demostrándose que los datos presentan distribución normal en ambas variables.

Después de este procedimiento estadístico, el cual es un supuesto que se debe cumplir para aplicar el estadígrafo correlación lineal de Pearson por tratarse de estadísticos paramétricos. A continuación, se muestran los resultados del programa estadístico Minitab.

\section{Método}

Tipo de correlación Pearson Filas utilizadas $\quad 316$

$\rho$ : correlación en parejas de Pearson

\section{Correlaciones}

\begin{tabular}{|l|r} 
& $\begin{array}{r}\text { Planificación } \\
\text { curricular }\end{array}$ \\
\hline Desempeño profesional & 0,525
\end{tabular}

Correlaciones en parejas de Pearson

\begin{tabular}{llrrr} 
& & \multicolumn{3}{c}{ IC de 95\% } \\
Muestra 1 & Muestra 2 & Correlación & para $\boldsymbol{\rho}$ & Valor p \\
\hline $\begin{array}{l}\text { Desempeño } \\
\text { profesional }\end{array}$ & Planificación & 0,525 & $(0,440 ;$ & 0,000 \\
& curricular & & $0,601)$ &
\end{tabular}


Según los resultados de la correlación lineal de Pearson se demuestra una correlación moderada entre la planificación curricular y el desempeño profesional de los egresados en educación (Valor $\mathrm{p}$ menor al nivel de significancia del 5\%), dando a conocer la influencia directa de la planificación curricular en la enseñanza universitaria, denotando que existe una estrecha articulación, pero debido al valor moderado de la correlación y los estadísticos descriptivos, es necesario revisar, evaluar y reajustar la planificación curricular, con un enfoque que responda a la articulación de lo teórico y práctico, incidiendo en este último.

Evaluando la regresión lineal en programa estadístico Minitab, se obtuvo:

Figura 3. Diagrama de dispersión de puntos con recta de ajuste de las variables planificación curricular y desempeño profesional de egresados en educación

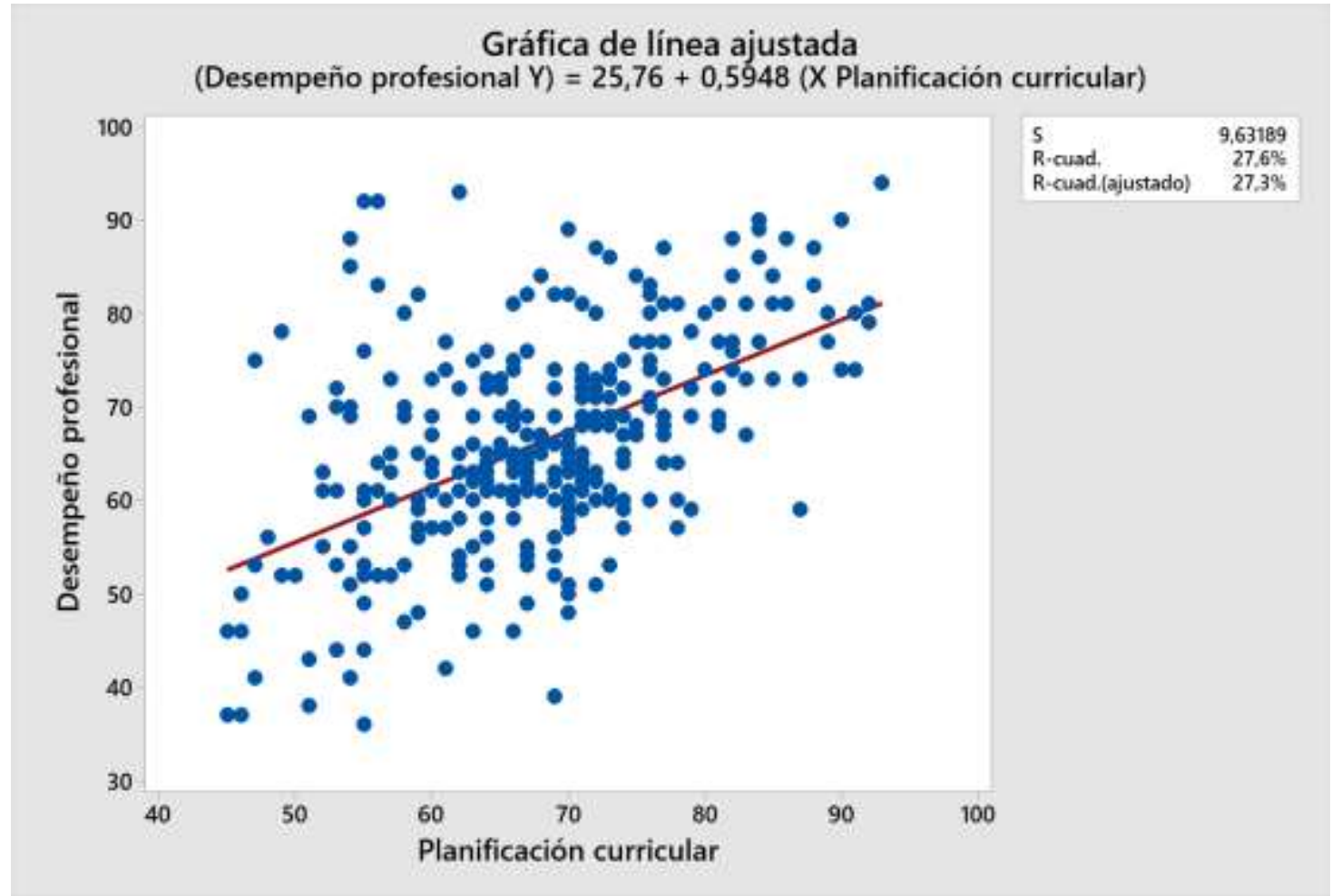

Nota: Datos procesados en el programa estadístico Minitab de un total de 316 egresados en educación.

La recta ajusta al diagrama de dispersión de puntos, demuestra que existe aglutinamiento de los datos de las variables planificación curricular y desempeño laboral, lo cual ya se ha validado con el estadígrafo correlación lineal de Pearson.

Resumen del modelo

\begin{tabular}{rrr} 
S & R-cuad. & $\begin{array}{r}\text { R-cuad. } \\
\text { (ajustado) }\end{array}$ \\
\hline 9,63189 & $27,56 \%$ & $27,33 \%$
\end{tabular}


Análisis de Varianza

\begin{tabular}{lrrrrr} 
Fuente & GL & SC & MC & F & P \\
\hline Regresión & 1 & 11082,0 & 11082,0 & 119,45 & 0,000 \\
Error & 314 & 29130,8 & 92,8 & & \\
Total & 315 & 40212,8 & & &
\end{tabular}

El análisis de la regresión lineal entre el desempeño profesional vs. la planificación curricular, la ecuación de regresión es:

(Desempeño profesional Y) = 25,76 + 0,5948 (X Planificación curricular)

Con la ecuación anterior es posible predecir un mejor desempeño profesional de los egresados en educación, para lo cual es necesario tener en cuenta una adecuada planificación curricular en el proceso de enseñanza universitaria. El R-cuadrado como valor predictivo, indica al 27,56\% de los egresados, estos suelen demostrar un desempeño profesional pertinente (de acuerdo a las exigencias del empleador), según la influencia el desarrollo de la planificación curricular durante la formación profesional del estudiante de la Escuela Profesional de Educación, en la diferencia del 72,44\% no es posible demostrar dicha influencia.

\subsection{Discusión}

En el desarrollo de la enseñanza universitaria desde sus comienzos hasta la actualidad está encaminada y regulada constantemente en el logro de las metas de programa, carrera o escuela profesional, en tal sentido, el currículo como producto de la historia social y humana, obedece a una construcción cultural, vinculada con la visión de la sociedad que se desea formar, en tanto las teorías curriculares reflejan la historia de las sociedades, en especial el papel que juega la educación en proceso de transformación y propagación de la sociedad. Incidiendo desde la perspectiva pedagógica el currículo fue concebido históricamente como: a) estructura organizada de conocimientos, basadas en las experiencias; b) plan; c) sistema y d) estrategia (Navarro, Pereira, Pereira, \& Fonseca, 2010, pp.214-215).

En ese entender, de acuerdo a los hallazgos del estudio, los futuros egresados de las escuelas profesionales de educación, programas o carreras afines, tienen el desafío de articular de mejor forma posible, con el desarrollo de competencias profesionales que se vinculen con el adecuado desempeño profesional y no solamente con un desempeño docente, centrado en la interacción docente y estudiante, sino también que esté involucrado con las necesidades de la comunidad educativa, proponiendo con creatividad, 
autonomía, trabajo en equipo y con sentido de innovación en la practica pedagógica y educativa en las instituciones educativas que llega a laborar.

El estudio ha evidenciado que los profesionales recientemente egresados presentan en su mayoría dificultades para acomodarse rápidamente en la práctica pedagógica y educativa de sus estudiantes, muchas veces sin alternativas practicas de solución, lo cual solamente presenta una mejoría considerable cuando llevan laborando por mas de 10 años en el desempeño profesional como educador. Esta situación se contrasta porque la planificación curricular, cuenta con la adecuación y estructura como se ha mencionado en la introducción del estudio, pero carece en su accionar, por la falta de aplicación del conocimiento aprendido o poner en práctica las capacidades, habilidades y destrezas adquiridas en el claustro universitario, llevando a la práctica lo que en teoría el estudiante ha aprendido, de tal forma que responda a un desempeño profesional eficiente con una correcta aplicación de los adquirido durante los 5 años de formación profesional. Ante esta exigencia la planificación curricular entra a tallar especialmente en el desarrollo de las practicas profesionales en instituciones educativas que formen parte de la alianza estratégica de las Facultades de Educación, asimismo implementar con recursos tecnológicos e infraestructuras modernas de laboratorios pedagógicos. En el caso de la Facultad de Educación y Ciencias de la Comunicación, cuenta con su propio laboratorio pedagógico (Institución Educativa Fortunato Luciano Herrera), el cual a pesar del esfuerzo de sus autoridades, aún continúa inconcluso la construcción de su local moderno, la administración y las compensaciones económicas son temas de gestión que requieren de la debida atención.

Consolidar un conjunto de rasgos distintivos de la imagen del futuro profesional en educación, supone que estos se exhiban pertinentemente luego de concluir los estudios profesionales, para lo cual es necesario, elaborar un plan de estudios que cuente con un sistema de seguimiento continuo para valorar la capacidad de respuesta en los futuros profesionales en educación.

\section{CONCLUSIONES}

Habiendo realizado los respectivos procesos de investigación, se logro explicar la influencia de la planificación curricular en la enseñanza universitaria respecto al eficiente desempeño profesional que todo profesional en educación debe demostrar, en tal sentido, solamente el 27,6\% de los profesionales en educación logran demostrar sus destrezas, 
habilidades y capacidades profesionales al momento de desplegar su autonomía profesional, apoyo a los estudiantes en su formación escolar, practicas pedagógicas reflexivas, relaciones con los padres de familia y relaciones profesionales entre directivos y docentes, donde la planificación curricular, esta orientado hacia la calidad educativa, pero, es recurrente indicar la falta de la articulación del conocimiento adquirido con su aplicación o llevada a la práctica.

El 72,4\% de los egresados presenta varias dificultades para desempeñarse eficientemente en la profesión de educador, esto debido a pesar de que la planificación curricular cuenta con los objetivos respectivos, la planificación de la enseñanza, el proceso de desarrollo de la enseñanza y la evaluación de aprendizajes, estos muchas veces resultan siendo más teóricos, olvidándose de la necesidad de llevarlo a la práctica, con lo cual sería posible afianzar las habilidades, capacidades y destrezas de los futuros egresados.

La ecuación resultante de la regresión lineal, (Desempeño profesional Y) $=25,76+$ 0,5948 (X Planificación curricular), denota la posibilidad de mejorar el desempeño profesional, esto implica un seguimiento permanente y una actualización constante de la planificación curricular, de acuerdo al avance vertiginoso del conocimiento, la tecnología y el conocimiento.

\section{LISTA DE REFERENCIAS}

Añorga, J. A. (1999). Paradigma educativo alternativo para el mejoramiento profesional y humano de los recursos laborales y de la comunidad: Educación Avanzada. La Habana: CENESEDA-ISPEJV.

Añorga, J. A. (2014). La educación avanzada y el mejoramiento profesional y humano. Varona. Revista Científico-Metodológica(58), 19-31.

Bastidas, F. A. (2019). Modelo holístico de planificación curricular para institutos y colegios universitarios de tecnología. Enfoques. Revista de Investigación en Ciencias de la Administración, 3(11), 202-2016.

Carrera, S. C., Parreño, J. d., \& Ayala, V. A. (2018). El desempeño profesional de los estudiantes universitarios en las prácticas pre-profesionales. Opuntia Brava, 9(1), 89-96.

Catalano, A. M., Avolio, S., \& Sladogna, M. G. (2004). Diseño curricular basado en normas de competencia laboral: Conceptos y orientaciones metodológicas (1 $\mathrm{ra}$ Edición ed.). Buenos Aires: BID - FOMIN. 
Compte, M. F. (2013). Las asignaturas optativas de formación profesional en la planificación curricular de pregrado. Universitas. Revista de Ciencias Sociales y Humanas, 11(18), 151-164.

González, M. I. (2006). Currículo basado en competencias: Una experiencia en educación universitaria. Educación y Educadores, 9(2), 95-117.

Hernández Sampieri, R., \& Mendoza Torres, C. P. (2018). Metodología de la Investigación: Las rutas cuantitativa, cualitativa y mixta. Ciudad de México: McGRAW-Hill Interamericana Editores, S.A. de C. V.

Johnson, R., \& Kuby, P. (2008). Estadística elemental: Lo esencial. México D.F.: Cengage Learning Editores, S.A.

Kaufman, R. A. (2011). Planificacion de sistemas educativos. Ideas báscias concretas (2da Edición ed.). México D.F.: Editorial Trillas.

Larraín, A. M., González, L. E., \& Espinosa, O. (Mayo de 2010). Incorporación del concepto de competencia en el diseño curricular universitario. Obtenido de researchgate.net: https://www.researchgate.net/profile/Luis-GonzalezFiegehen/publication/275275496_Incorporacion_del_concepto_de_competencia _en_el_diseno_curricular_universitario_2010-

15/links/5536deac0cf2058efdea9562/Incorporacion-del-concepto-decompetencia-en-el-diseno

Litwin, E. (2006). El currículo universitario: Perspectivas teóricas y metodológicas para el análisis y el cambio. Revista Educación y Pedagogía, 18(46), 25-31.

Medrano, H., \& Molina, S. A. (2010). Desempeño profesional de docentes del Siglo XXI. Congreso Iberoamericano de Educación (págs. 1-22). Buenos Aires: Instituto de Investigación, Innovación y Estudios de Posgrado para la Educación.

Mejía, E. (2011). El proceso de elaboración del currículo para la formación profesional universitaria. Investigación Educativa, XV(28), 109-127.

Moscoso, F., \& Hernández, A. (2015). La formación pedagógica del docente universitario: un reto del mundo contemporáneo. Revista de Educación Superior, 140-154.

Navarro, Y., Pereira, M., Pereira, L., \& Fonseca, N. (2010). Una mirada a la planificación estratégica curricular. Telos, 12(2), 202-216. 
Salas, R. S., Hatim, A., \& Rey, R. (1997). Sistema de monitoreo y control de calidad de la competencia y el desempeño profesional. Educación Médica Superior, 11(1).

Tobón, S. (2008). La formación basada en competencias en la educación superior: El enfoque complejo. México: Universidad Autónoma de Guadalajara.

Tobón, S., Pimienta, J. H., \& García, J. A. (2010). Secuencias didácticas: Aprendizaje y evaluación de competencias (1ra Edición ed.). México D.F.: Pearson Educación de México, S.A. de C.V.

Zabalza, M. Á. (2000). Los planes de estudio en la universidad. Algunas reflexiones para el cambio. Revista Fuentes(1), 27-68. 